\title{
Long-term disease free survival of gastric mixed adenoneuroendocrine carcinoma treated with multimodality therapy: A case report
}

\author{
GUANGJIAN YANG ${ }^{1}$, DAN LI $^{2}$, FANGCHAO ZHENG ${ }^{3}$ and LIN YANG $^{1}$ \\ ${ }^{1}$ Medical Department of National Cancer Center/Cancer Hospital, Chinese Academy of Medical \\ Sciences and Peking Union Medical College, Beijing 100021; ${ }^{2}$ Department of Pathology, Shengjing \\ Hospital of China Medical University, Shenyang, Liaoning 110004; ${ }^{3}$ Department of Oncology, \\ Shouguang Hospital of Traditional Chinese Medicine, Weifang 262700, P.R. China
}

Received August 16, 2017; Accepted December 11, 2017

DOI: $10.3892 / \mathrm{mco} .2018 .1594$

\begin{abstract}
Gastric mixed adenoneuroendocrine carcinoma, a rare malignant type of stomach tumor, is composed of components of adenocarcinoma and neuroendocrine tumor. This type of neoplasm usually has a poor prognosis since both components are malignant. A 65-year-old male presented with norexia, epigastric distention, abdominal and lumbar pain. Contrast computed tomography scan of the abdomen verified multiple lesions merged into a large mass. Histolopathological examination of the specimen from digestive endoscopy was verified to be gastric mixed adenoneuroendocrine carcinoma. The patient received a multimodality treatment of chemotherapy, surgical resection with radiotherapy and still remains alive with no evidence of metastasis or recurrence for over 5 years. The authors suggest that multimodality therapy may be beneficial and necessary to effictively treat mixed adenoneuroendocrine carcinoma.
\end{abstract}

\section{Introduction}

According to the most recent classification of the World Health Organization in 2010, mixed carcinomas containing an exocrine and a neuroendocrine component, with one component exceeding $30 \%$, are designated as mixed adenoneuroendocrine carcinomas (MANECs) (1). Neuroendocrine neoplasm of the

Correspondence to: $\mathrm{Dr}$ Lin Yang, Medical Department of National Cancer Center/Cancer Hospital, Chinese Academy of Medical Sciences and Peking Union Medical College, No. 17 Panjiayuannanli, Chaoyang, Beijing 100021, P.R. China

E-mail: lyang69@sina.com

Abbreviations: MANEC, mixed adenoneuroendocrine carcinoma; NET, neuroendocrine tumor; NEC, neuroendocrine carcinoma

Key words: adenoneuroendocrine carcinoma, long-term disease free survival, multimodality therapy, MANEC, gastric cancer stomach were divided into three categories: Neuroendocrine tumor (NET), neuroendocrine carcinoma (NEC) and mixed adenoneuroendocrine carcinoma (MANEC). In practice, there is much variation in the percentage of components between adenocarcinoma and neuroendocrine tumor in stomach neoplasm. However, MANEC consists of both components with at least $30 \%$ of either component. Those which fit in the criteria of MANEC are rare and only case report can be found in the literature (2). The epidemiology of MANEC has not yet been described. This kind of neoplasm usually has a poor prognosis since both components are malignant. Here we report a patient with a locally advanced unresectable gastric MANEC who received multimodality treatment including chemotherapy, surgical resection and radiotherapy, still remains alive with no evidence of metastasis or recurrence at more than five years so far.

\section{Case report}

In May 2012, a 65-year old male was admitted to our hospital, presented with anorexia, epigastric distention, abdominal and lumbar pain and weight loss over a one-month period. His past medical history included a chronic gastritis and he had undergone appendectomy 31 years earlier. Physical examination showed no abnormalities.

An ultrasound revealed a substantial mass in the upper abdominal cavity. A subsequent contrast CT scan of the abdomen confirmed that multiple lesions located in the lesser curvature of the stomach, hepatic hilar region, mesenteric root, retroperitoneal region and above the head of the pancreas. All of these lesions merged into a large mass, measuring approximately $5.5 \times 4.8 \mathrm{~cm}$ (Fig. 1A and B). Upper gastrointestinal endoscopy showed an ulcerous lesion at lesser curvature of stomach with protruded ring dike-like mucous membrane. Endoscopic ultrasonography revealed that the tumor was located at the mucosal and submucosal layer. The histolopathological examination from the ulcerous lesion and ultrasound-guided fine needle aspiration of one of the lesions revealed MANEC (Fig. 2A), and neuroendocrine markers for chromogranin A (Fig. 2B) and synaptophysin (Fig. 2C) were 

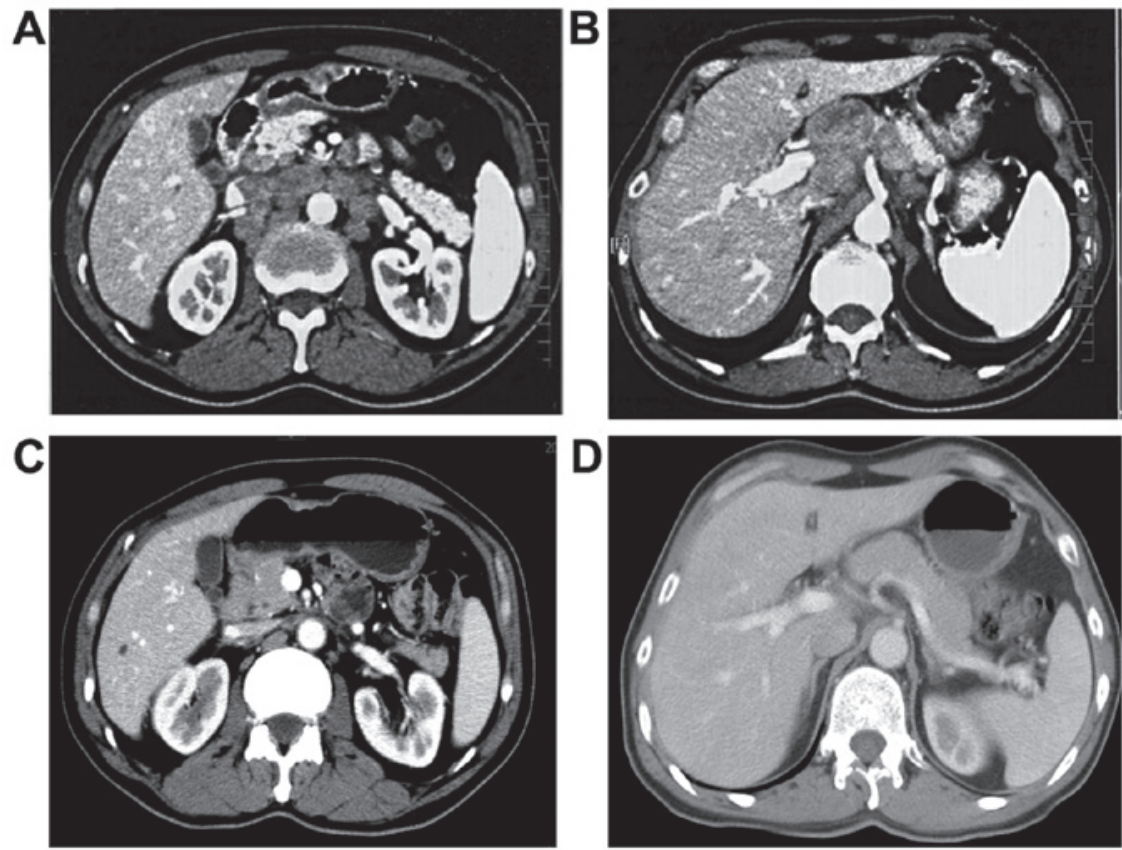

Figure 1. Multiple lesions located in the hepatic hilar region, mesenteric root, retroperitoneal region and above the head of the pancreas merged into a large mass before treatment (A and B) and shrunk rapidly after four cycles of chemotherapy (C and D).
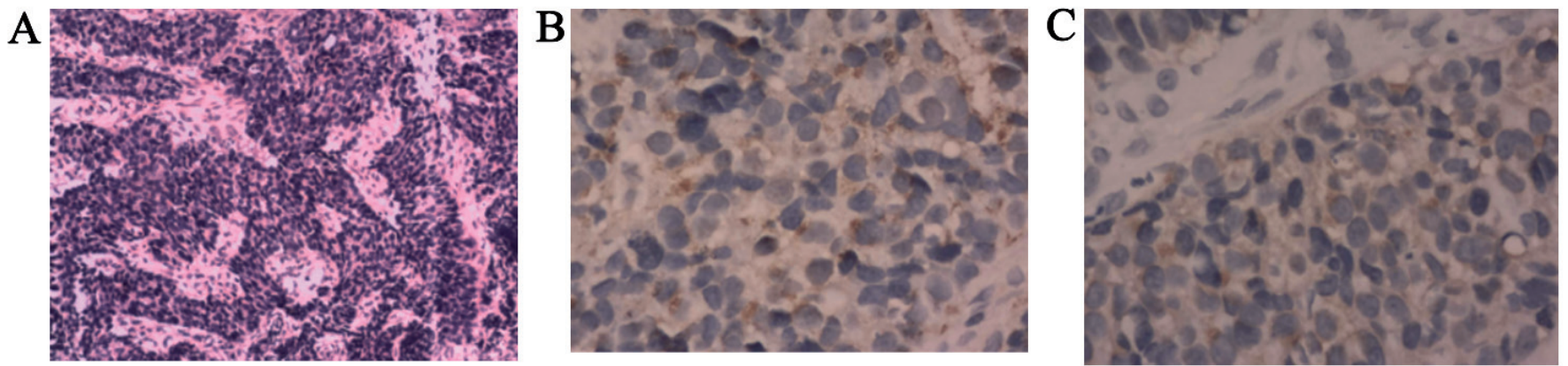

Figure 2. Microscopic findings of gastric mixed carcinoma, containing an exocrine and a neuroendocrine component. (A) H\&E staining and (B and C) immunohistochemical staining showing immunoreactivity for (B) chromogranin A and (C) synaptophysin; magnification, x400.

positive in the neuroendocrine component only and negative in the adenocarcinoma component. The staging chest X-ray showed no thoracic disease.

On the basis of tumor biology and the disease stage, the patient was not a candidate for surgery, and systemic cytotoxic chemotherapy was scheduled from May 26 to August 14 in 2012, four cycles of chemotherapy consisting of VP16, cisplatin and TS-1 (repeated every 21 days) were administered to the patient. Contrast CT scan afterwards showed that the thick of gastric wall had little change but the multiple enlarged lymph nodes shrunk rapidly and significantly (Fig. 1C and D). The main adverse effects of chemotherapy included grade 3 leukopenia, grade 2 neutropenia, grade 2 anorexia, grade 1 nausea and grade 1 vomiting. Related guidance advocates that all gastric small cell carcinoma should be treated with oncological resections without considering grade and stage (3). Based on the excellent response, on September 10, 2012, the patient undergone open distal gastrectomy with D2 lymphadenectomy. But the number 16 lymph nodes were not dissected. Gross examination of the surgical specimen revealed an ulcerous lesion measuring $0.9 \times 0.6 \times 0.3 \mathrm{~cm}$ located at anterior of antrum near lesser curvature. Histolopathological examination of the surgical specimen showed that there was no neuroendocrine component. Only a little degenerated adenocarcinoma cells remained in the mucosa, which invaded muscularis mucosae and were infiltrated with large amount of inflammatory cells. No metastasis was revealed among the checked 28 lymph nodes.

Four weeks after surgical resection, the patient received additional two cycles of postoperative chemotherapy with the same regimen. The tolerability was well. The main adverse effects included grade 1 neutropenia, grade 1 decline of hemoglobin and grade 1 nausea.

Considering the high rate of local recurrence, combined treatment with radiation therapy was adopted. From December 24 to January 25 in 2013, concurrent chemo-radiotherapy was administered. Lymph node stations in the radiation fields included the retroperitoneal lymph nodes with No. 7, 8, 12, 13, 14 and 16 lymph nodes. Especially, the radiation focus was on No. 16 lymph nodes to give clinical target volume. The planning target volume dosage was $45 \mathrm{~Gy}$, 
which was delivered in 25 fractions within 35 days. PTV1 was $56 \mathrm{~Gy} / 25 \mathrm{f} / 35 \mathrm{~d}$ with concurrent oral capecitabine. The main adverse effects included grade 1 decline of hemoglobin, grade 2 anorexia, grade 1 nausea and grade 1 fatigue.

After completion of all above therapy, the patient undergone regular follow-up every 4 to 6 months, including CT scan of chest, abdomen, pelvis and blood chemistry. Until the last follow up in May of 2017, the patient has been alive for 5 years since diagnosis without recurrence or metastasis.

\section{Discussion}

There is a wide spectrum of combinations of exocrine and neuroendocrine components in the stomach neoplasm. However, according to the 2010 WHO grading system, MANECs are characterized by only those neoplasms in which each component represents at least $30 \%$ of the lesion. But the $30 \%$ cut-off is not based on enough data on the prognostic significance of the neuroendocrine component. In Park et al study (4), $10 \%$ cut-off may be more clinically useful and may serve as an informative parameter for predicting patient outcome. All eligible patients had received gastrectomy and were staged I-III. The 5-year OS rates for NEC, MANEC and gastric carcinoma with neuroendocrine differentiation were 59.1, 53.5 and $16.7 \%$, respectively. However, the 5-year OS in patients with gastric carcinomas without neuroendocrine morphology is $85.2 \%$. Although the early detection and treatment of gastric tumors has increased in accordance with the progression of endoscopic techniques, the early detection of MANEC is extremely rare. Even in the early stage of the disease, the neuroendocrine carcinoma component is detected in the deeper portion of the mucosal or submucosal layers, and can only be diagnosed after resection of the tumor.

Until now, the research for MANEC prognosis and treatment is limited, also because MANEC in the stomach is extremely rare, there is no recommended therapeutic treatment strategy.

Surgical resection is indicated in patients without metastasis. However, curative surgery is probably possible in $<30 \%$ of all NET patients and recurrence is common (5). When curative surgery is not possible, locoregional resective surgery was associated with increased survival on crude and multivariate analysis (6).

As the high risk of metastasis and recurrence rate of MANEC, chemotherapy has been reported to be the treatment of choice, which had improved the median survival to a range of 6 to 12 months, with occasional long-term survival (7). So far, the chemotherapy regimen of MANECs is not standardized. Owing to the effective chemotherapeutic regimen with a combination of etoposide and cisplatin in treating small cell lung cancer, the first study explored infusional cisplatin and etoposide among 45 patients with metastatic NECs (of whom 18 had PD tumors) between 1987 and 1990. In this study, a response rate of $67 \%$ was reported in patients with PD NECs, with response duration of 8 months and a median survival of 19 months (8). Alternative regimens substituting carboplatin for cisplatin or irinotecan for etoposide have been validated in metastatic small-cell lung cancer and are therefore thought to be acceptable options for management of PD NECs (9). Based on the treatment paradigm for limited-stage small-cell lung cancer, first-line systemic chemotherapy with a platinum agen and etoposide (cisplatin or carboplatin and etoposide for 4-6 cycles) is recommended for most patients with metastatic-stage disease (10). Especially, chemotherapy combined with radiation can be considered patients with MANECs, particularly when surgical resection is difficult.

Although the biological similarity between small cell carcinoma of the lung and gastric neuroendocrine carcinoma is controversial, the EP regimen (combination of etoposide and cisplatin), which is used for small cell carcinoma of the lung, is the most widely used treatment in neuroendocrine carcinoma. For this case, we selected TS-1, cisplatin, etoposide and achieved a good partial response. The postoperative pathology showed that only little adenocarcinoma component remained. Although the role of radiation for MANEC is unclear, as with small cell lung cancer, we think MANEC is sensitive to radiation, so we give the patient local radiation to reduce the risk of local recurrence. Several trials and meta-analyses have shown that radiotherapy combined with chemotherapy could improve the curative effects, provide regional control and do help to subsequent long term survival in isolated cases (11).

After surgery resection with 6 cycles of perioperative chemotherapy of EP regimen and consolidated radiotherapy to the tumor remained region, the patient has a long term disease free survival. We suggest that multimodality therapy can be beneficial and necessary to the MANEC.

\section{Acknowledgements}

Not applicable.

\section{Consent for publication}

Written informed consent was obtained from the patient for publication of this case report and any accompanying images.

\section{Competing interests}

The authors declare that they have no competing interests.

\section{References}

1. Kitajima T, Kaida S, Lee S, Haruta S, Shinohara H, Ueno M, Suyama K, Oota Y, Fujii T and Udagawa H: Mixed adeno(neuro) endocrine carcinoma arising from the ectopic gastric mucosa of the upper thoracic esophagus. World J Surg Oncol 11: 218, 2013.

2. Gurzu S, Kadar Z, Bara T, Bara T Jr, Tamasi A, Azamfirei L and Jung I: Mixed adenoneuroendocrine carcinoma of gastrointestinal tract: Report of two cases. World J Gastroenterol 21: 1329-1333, 2015.

3. Basuroy R, Srirajaskanthan R, Prachalias A, Quaglia A and Ramage JK: Review article: The investigation and management of gastric neuroendocrine tumours. Aliment Pharmacol Ther 39: 1071-1084, 2014

4. Park JY, Ryu MH, Park YS, Park HJ, Ryoo BY, Kim MG, Yook JH, Kim BS and Kang YK: Prognostic significance of neuroendocrine components in gastric carcinomas. Eur $\mathrm{J}$ Cancer 50: 2802-2809, 2014.

5. Janson ET, Sorbye H, Welin S, Federspiel B, Grønbæk H, Hellman P, Ladekarl M, Langer SW, Mortensen J, Schalin-Jäntti C, et al: Nordic guidelines 2014 for diagnosis and treatment of gastroenteropancreatic neuroendocrine neoplasms. Acta Oncol 53: 1284-1297, 2014.

6. Norlén O, Stålberg P, Öberg K, Eriksson J, Hedberg J, Hessman O, Janson ET, Hellman P and Âkerström G: Long-term results of surgery for small intestinal neuroendocrine tumors at a tertiary referral center. World J Surg 36: 1419-1431, 2012. 
7. Richards D, Davis D, Yan P and Guha S: Unusual case of small cell gastric carcinoma: Case report and literature review. Dig Dis Sci 56: 951-957, 2011

8. Moertel CG, Kvols LK, O'Connell MJ and Rubin J: Treatment of neuroendocrine carcinomas with combined etoposide and cisplatin. Evidence of major therapeutic activity in the anaplastic variants of these neoplasms. Cancer 68: 227-232, 1991.

9. Hanna N, Bunn PA Jr, Langer C, Einhorn L, Guthrie T Jr, Beck T, Ansari R, Ellis P, Byrne M, Morrison M, et al: Randomized phase III trial comparing irinotecan/cisplatin with etoposide/cisplatin in patients with previously untreated extensive-stage disease small-cell lung cancer. J Clin Oncol 24: 2038-2043, 2006.
10. Strosberg JR, Coppola D, Klimstra DS, Phan AT, Kulke MH, Wiseman GA and Kvols LK: North American Neuroendocrine Tumor Society (NANETS): The NANETS consensus guidelines for the diagnosis and management of poorly differentiated (high-grade) extrapulmonary neuroendocrine carcinomas. Pancreas 39: 799-800, 2010.

11. Brenner B, Tang LH, Shia J, Klimstra DS and Kelsen DP: Small cell carcinomas of the gastrointestinal tract: Clinicopathological features and treatment approach. Semin Oncol 34: 43-50, 2007. 